\title{
Contextes de formation formel, non formel ou informel: développement de compétences de direction d'école de langue française au Canada
}

\section{Claire IsaBelle, Andréanne Gélinas Proulx et Hélène Meunier}

Pour parfaire les compétences des nouvelles directions d'école, nous constatons l'émergence de programmes de formation proposés par des universités, des districts scolaires, etc. Le but de notre étude est d'identifier les contextes de formation formel, non formel ou informel qui ont le plus aidé les nouvelles directions d'école dans le développement de leurs compétences d'une part, et ceux qui seraient mieux à même de les aider à l'avenir. Dans le cadre de cette recherche qualitative, 101 acteurs-trices de l'éducation ont été interrogé-es. Les résultats montrent que les trois contextes de formation (formel, non formel et informel) semblent avoir contribué au développement des compétences des nouvelles directions, alors que le contexte non formel, et plus particulièrement les ateliers et le soutien du district scolaire, s'avère être celui pouvant le plus aider les nouvelles directions à développer leurs compétences dans le futur.

\section{Int roduction}

Les études le confirment: le leadership de la direction d'école fait une différence! En effet, des études soulignent l'apport essentiel de la direction d'école pour instaurer un climat organisationnel qui encourage chez le corps enseignant: la collaboration, le développement professionnel, les pratiques pédagogiques renouvelées voire l'épanouissement afin de favoriser chez l'élève son plein potentiel (Seashore Louis, Dretzkekl \& Wahlstrom, 2010; Lapointe \& Gauthier, 2005). Ainsi dans la foulée de préparer les directions et futures directions d'école à la gestion complexe d'une école et à exercer un leadership qui garantit la réussite scolaire des élèves, de plus en plus de formations institutionnalisées sont exigées et des formations en milieu de travail sont proposées. En effet, plusieurs ministères d'Éducation des provinces et territoires canadiens exigent un minimum d'un diplôme d'études supérieures (maîtrise et diplôme d'études supérieures spécialisées) pour obtenir un poste à la direction d'une école canadienne (IsaBelle et al., 2008). 


\section{Apport des études récentes sur une formation plus adaptée}

En 1999, aux États-Unis, la National Association of State Boards of Education (NASBE) signale la qualité inférieure des programmes de certification à la direction d'école (Fossey \& Shoho, 2006). Dans la même veine, certaines études confirment que la formation universitaire offerte aux étudiant-e-s en principalat ne les prépare pas adéquatement pour faire face aux réalités de l'école (DarlingHammond, LaPointe, Meyerson \& Orr, 2007; Levine, 2005; Young, Crow, Orr, Ogawa \& Creighton, 2006). Ces programmes de formation universitaire ou ces formations offertes dans un contexte formel manquent de rigueur et leurs contenus sont jugés parfois trop théoriques et peu reliés à la pratique, ou trop pratiques et pas assez théoriques (Lapointe \& Langlois, 2004). Qui plus est, un rapport de l'Organisation de coopération et de développement économiques (OCDE, 2008) stipule qu'il est ardu de remplacer les chefs d'établissement qui vont à la retraite, entre autres, à cause de l'hésitation des candidats potentiels qui soulignent avoir reçu une préparation insuffisante pour occuper les postes vacants. En plus, un remplacement important des directions et ce jusqu'en 2020 est prévu selon les statistiques canadiennes (Service Canada, 2012).

Des études démontrent qu'il est possible d'offrir des formations de qualité et utiles aux directions et futures directions d'école si des caractéristiques spécifiques sont respectées (Pounder, 2011). Par exemple, les directions d'école qui complètent un programme de formation dans une institution qui effectue de la recherche ou qui offre des programmes de niveau doctorat peuvent améliorer la qualité de l'enseignement du personnel (Fuller, Young \& Baker, 2011). Dans la même foulée, selon Sanzo, Myran et Clayton (2011), quand une formation à la direction est offerte conjointement par une faculté universitaire et les districts scolaires ${ }^{1}$, les candidat-e-s au principalat peuvent plus facilement marier la théorie à la pratique. Ainsi constatons-nous l'émergence de programmes de formation qui sont conjointement offerts par des universités et des associations professionnelles ou des agences non gouvernementales, etc. (Walker, Bryant \& Lee, 2013). Dans le même sens, les résultats de deux études canadiennes montrent que les directions d'école ont bénéficié des contextes formel et informel pour faciliter leur insertion professionnelle (Cattonar et al., 2007) et semblent préférer le contexte informel au contexte formel pour développer leurs compétences (IsaBelle et al. 2008). Ces deux études seront abordées dans la partie de la problématique.

Devant les différentes offres de formation, il devient crucial d'analyser les contextes de formation qui favorisent le développement des compétences des nouvelles directions d'école de langue française au Canada, car étudier ces contextes de formation permettra de mieux comprendre et répondre éventuellement à leurs besoins afin qu'elles puissent accomplir leurs tâches efficacement.

Dans les prochaines sections, le cadre conceptuel, la problématique, la méthodologie ainsi que les résultats seront présentés. 


\section{Cadre conceptuel}

Dans cette section, nous aborderons les contextes de formation et le concept de compétences.

\section{Contextes de formation}

Force est de constater que nous ne retrouvons pas un consensus quant à la définition des différents contextes de formation. Toutefois, les travaux convergent:

Tout le monde apprend partout et tout le temps. Le constat n'est plus original et il semble y avoir consensus sur cette qualité qu'ont les individus d'accumuler savoirs, savoir-faire et/ou compétences tout au long de leur vie, même bien au-delà des apprentissages organisés dans un contexte formel comme l'école, l'université ou la formation professionnelle structurée. (Werquin, 2010, p. 16)

Or, comme le souligne l'auteur, les apprentissages réalisés dans les contextes non formels et informels sont aussi considérables, c'est pourquoi les contextes formels ne peuvent plus être reconnus comme les seuls engendrant des apprentissages. De façon récurrente, nous retrouvons dans la littérature trois contextes de formation: formel, non formel et informel.

Le contexte formel de formation est lié aux systèmes d'éducation et de formation régis par l'État. Ce contexte conduit à des enseignements qui mènent à l'obtention de diplômes ou de titres décernés par l'État (Marchand, 1997; Merriam, Caffarella \& Baumgartner, 2009). Pour Schugurensky (2000), ce contexte de formation est propédeutique, c'est-à-dire que chaque niveau de formation est prérequis pour atteindre un autre niveau de formation et il est nécessaire de les compléter afin d'obtenir un diplôme. Par exemple, dans ce contexte nous retrouvons l'enseignement secondaire, collégial et universitaire, soit pour une formation initiale ou supérieure. Dans le contexte formel, contrairement à la définition de Werquin (2010), nous ne considérons pas la formation offerte par l'employeur ou par toutes autres organisations dont la principale activité n'est pas l'enseignement qui mène à un diplôme reconnu par l'État. Pour nous, cette formation s'inscrit dans un contexte de formation non formel.

Le deuxième contexte de formation, non formel, renvoie aux activités de formation qui n'aboutissent pas à un diplôme ou à une qualification reconnue. Les activités sont d'une durée très limitée et suivies sur une base volontaire (Schugurensky, 2000). Livingston (1999) surnomme ce contexte de formation: la formation continue. Par exemple, nous retrouvons les ateliers de formation dispensés par les districts scolaires et les associations professionnelles. Nous incluons aussi les colloques et les rencontres-ateliers planifiés par le ministère de l'Éducation de même que le coaching et les communautés d'apprentissage professionnelles mis en place, entre autres, par les districts scolaires. 
Finalement, le contexte de formation informel représente toutes activités organisées par l'individu pour parfaire ses connaissances et ses compétences à l'extérieur du cadre formel d'une institution accréditée ou du cadre non formel d'un organisme éducationnel ou autre (Livingston, 1999). Par exemple, nous retrouvons l'utilisation des médias, les échanges entre collègues, la lecture, l'implication bénévole dans des organisations de la communauté. Plusieurs auteurs caractérisent cette formation selon l'intentionnalité et la conscience ou non de l'apprentissage. Ainsi Schugurensky (2000) subdivise-t-il le contexte de formation informel en trois types: l'apprentissage autodirigé (self-directed learning), l'apprentissage fortuit (incidental learning), la socialisation ou l'apprentissage tacite (socialization, as tacit learning). Ce dernier type rejoint la définition du contexte de formation informel de Merriam et al. (2009). Dans l'étude d'IsaBelle et al. (2008), deux sous-catégories ont émergé des données. La catégorie Contexte informel 1 comprend les pratiques de formation reliées à la discussion, telles qu'en discutant avec des directions d'école, des enseignant-e-s et des ami-e-s. La catégorie Contexte informel 2 inclut l'apprentissage par des lectures personnelles, par "essais et erreurs», soit "sur le tas» en milieu de travail ou en participant à des activités bénévoles.

Finalement, les différents contextes de formation permettent de développer des compétences comme le mentionne Werquin (2010), mais qu'entend-on par compétence?

\section{Compétences}

Roegiers et De Ketele (2000) mentionnent que «la compétence est la possibilité, pour un individu, de mobiliser de manière intériorisée un ensemble intégré de ressources en vue de résoudre une famille de situations-problèmes [toutefois] c'est toujours grâce à ses ressources internes que la personne peut mobiliser des ressources externes» (p. 66). Laurier (2005) précise que les ressources d'une compétence sont «une combinaison de connaissances, d'habiletés et d'attitudes» produisant «un savoir-agir» (p. 16) qui, si un individu l'intègre à sa pratique, fera de lui un professionnel compétent dans les fonctions qu'il exerce. À cet effet, Meirieu (2005) mentionne: «Pour qu'une compétence soit transférable, il faut que l'apprenant sache reconnaître les situations nouvelles où elle peut s'appliquer» (p. 25). Ainsi, développer des compétences nécessite des occasions de formation et celles-ci peuvent être variées et provenir de contextes formels, non formels et informels.

\section{Problématique et questions de recherche}

Dans cette section, nous présentons les résultats de deux études de nature quantitative qui ont été menées auprès de directions d'école au Canada en lien avec les trois contextes de formation. 


\section{Contexte de formation formel}

Une première enquête par questionnaire a été réalisée en 2005 auprès de 2144 directions d'école primaire et secondaire de langues française et anglaise (Cattonar et al., 2007). Cette enquête visait à étudier le métier des directions d'école en partant des représentations subjectives que celles-ci ont de leur métier tout en tenant compte des conditions objectives dans lesquelles se déroulent leurs expériences professionnelles. Le questionnaire comprend plusieurs sections, dont une portant sur l'insertion et le développement professionnels des directions d'école. Les résultats démontrent que $89,5 \%$ des directions indiquent que leur formation antérieure leur a été utile. Bien qu'il ne soit pas précisé de quel contexte de formation antérieure il est question, nous pouvons croire qu'il s'agit de leur formation universitaire. De plus, les directions qui considèrent que leur formation antérieure leur a été utile au début de leur carrière possèdent une perception plus positive de leurs compétences. Les directions qui ont complété un diplôme de deuxième cycle (maîtrise) sont plus nombreuses à considérer que, lorsqu'elles ont commencé leur emploi, elles possédaient les compétences pour les aspects administratifs de leur travail et étaient préparées pour assumer leurs fonctions.

Une deuxième étude pancanadienne d'IsaBelle et al. (2008) a été menée auprès de 213 directions et directions adjointes d'école de langue française. Le but de l'étude consistait à identifier les contextes de formation que les directions d'école francophones en milieu minoritaire préferent, à partir d'un questionnaire comprenant, entre autres, l'énumération de 14 activités. Les activités se classifiaient selon les trois contextes de formation que nous avons présentés dans le cadre conceptuel. Les directions devaient indiquer leur préférence selon une échelle de type Likert à cinq niveaux (N'aime pas du tout à Aime beaucoup). Les résultats des analyses ANOVA indiquent que le contexte formel de formation est le moins apprécié par les directions d'école de l'étude. Pour obtenir des données plus fines, les participant-e-s ont été divisé-e-s en deux groupes: les directions d'école ayant cinq ans et moins d'expérience et les directions d'école ayant six ans et plus d'expérience. Les analyses du test- $T$ n'indiquent aucune différence significative entre les deux groupes de directions quant à leur préférence pour le contexte formel de formation.

En somme, d'après les deux études quantitatives, nous constatons que même si le contexte de formation formel semble peu apprécié (IsaBelle et al., 2008), il demeure qu'il permet aux directions d'école d'être préparées pour assumer leurs fonctions et pour répondre aux besoins administratifs de leur travail au début de leur carrière (Cattonar et al., 2007). Ainsi une forte majorité des directions d'école le qualifie-t-elle d'utile.

\section{Contexte de formation non formel}

De l'enquête réalisée par Cattonar et al. (2007) se dégagent aussi des résultats pertinents quant au contexte de formation non formel. D'une part, 74,7\% 
des directions ayant participé à l'étude affirment être en accord avec l'énoncé J'ai reçu l'aide de mes supérieurs. D'autre part, afin de connaitre les différentes mesures d'accueil, de soutien et d'accompagnement que les directions ont reçues au début de leur carrière, une série de six mesures leur a été proposée. Les résultats démontrent que dans l'ensemble certaines directions d'école ont bénéficié de mesures telles que, J'ai pu profiter d'un perfectionnement approprié (58,5\%); J'ai pu participer à un réseau d'entraide entre directeurs (53,3\%); Il y a eu des activités d'accueil (34,5\%); J'ai pu compter sur une personne-ressource désignée (34,7\%); J'ai pu participer à un groupe de soutien (32,9\%) et J'ai bénéficié d'un parrainage ou mentorat $(38,2 \%)$. Également, à la question ouverte avez-vous d'autres mesures d'accueil, de soutien et d'accompagnement à mentionner, près de $12 \%$ des directions ont indiqué avoir bénéficié d'autres types de soutien, tels qu'avoir une expérience préalable dans une fonction connexe, faire des lectures, assister à des conférences, participer à des ateliers, faire appel à leurs réseaux pour trouver de l'aide. Selon les auteurs, ces mesures semblent informelles et d'initiatives personnelles. En fait, nous avons peu d'information quant aux différentes mesures évaluées. Par exemple, de quelles activités d'accueil ou de perfectionnement les directions d'école ont-elles bénéficiées? De plus, le réseau d'entraide et le groupe de soutien étaient-ils formés par leur district scolaire ou étaient-ils formés de façon spontanée entre les directions d'école? Selon les auteurs :

Toutes les mesures d'accueil, de soutien et d'accompagnement des directeurs sont fortement associées à l'énoncé selon lequel ils ont reçu de l'aide de leurs supérieurs lorsqu'ils ont assumé pour la première fois la direction d'une école. On peut supposer qu'il s'agit d'énoncés équivalents, c'est-à-dire que les différentes mesures d'accueil, de soutien ou d'accompagnement des directeurs constituent justement l'aide qu'ils ont reçue de la part de leurs supérieurs. (p. 418)

C'est pourquoi nous catégorisons la majorité de ces résultats dans le contexte non formel de formation, car ils semblent s'inscrire dans une planification du district scolaire.

Selon l'étude pancanadienne d'IsaBelle et al. (2008), les analyses du test-T indiquent des différences significatives entre les deux groupes de directions quant au contexte non formel. En effet, comparativement aux directions expérimentées, les nouvelles directions affirment apprécier développer leurs compétences, par ordre d'appréciation, en suivant des formations offertes par leur district scolaire, en participant à des activités planifiées par le district scolaire et l'école telles que le mentorat, les communautés d'apprentissage professionnelles, des groupes de développement professionnel ainsi qu'en suivant des formations offertes par leur ministère de l'Éducation. De toutes les activités évaluées, assister à des conférences hors de la province et du territoire semble être l'activité la moins appréciée tant par les nouvelles directions que les directions d'école expérimentées. 
En somme, les résultats de l'enquête de Cattonar et al. (2007) suggèrent que les directions d'école ont bénéficié de l'aide de leurs supérieurs, d'un perfectionnement approprié et d'un réseau d'entraide entre directions pour faciliter leur insertion professionnelle. Toutefois, les items en lien avec le contexte de formation non formel évalués dans l'enquête de Cattonar et al. sont peu précis. L'étude d'IsaBelle et al. (2008) démontre que les nouvelles directions affirment apprécier suivre des formations offertes par leur district scolaire et participer à des activités coordonnées par le district scolaire et l'école, telles que le mentorat, etc. Bref, il semble que le contexte non formel est utile et aimé des directions d'école pour développer des compétences, mais certaines limites surtout terminologiques des recherches présentées demandent que d'autres études soient entreprises sur le sujet.

\section{Contexte informel}

Selon l'enquête de Cattonar et al. (2007), 96,1\% des directions d'école affirment être en accord avec l'énoncé J'ai appris cette fonction sur le tas. De plus, tel que mentionné plus haut, à la question portant sur les différentes mesures d'accueil, de soutien et d'accompagnement que les directions d'école ont reçues au début de leur carrière, près de $12 \%$ des répondant-e-s ont indiqué avoir bénéficié d'autres mesures. Certaines relèvent selon nous du contexte informel de formation, telles qu'avoir une expérience préalable dans une fonction connexe, faire des lectures, faire appel à leurs réseaux pour trouver de l'aide.

Dans l'étude d'IsaBelle et al. (2008), le test-T indique de façon significative pour la première sous-catégorie, contexte informel 1 , que les nouvelles directions apprécient davantage développer des compétences en discutant avec leurs pairs, des enseignant-e-s et des ami-e-s que les directions plus expérimentées. En ce qui a trait à la deuxième sous-catégorie, contexte informel 2 , les résultats du test-T ne sont pas significatifs. Cependant, les moyennes élevées (3,34 et + sur 5) aux énoncés montrent que la majorité des directions des deux groupes semble apprécier développer leurs compétences par des lectures et par essais-erreurs, soit sur le tas.

À la lumière des résultats de ces deux études quantitatives pancanadiennes, tout indique que nous en savons peu sur les contextes de formation des directions d'école et qu'une recherche qualitative nous permettrait d'approfondir cet objet de recherche. "Nous ne savons pas exactement quelles formes devraient prendre les programmes en administration éducationnelle formels et quels contenus, quelles méthodes pédagogiques et quelles structures devraient-ils employer» (LaMagdeleine, Maxcy, Pounder \& Reed, 2009, p. 130). A fortiori, Livingston (1999) et Merriam et al. (2009) soulignent que peu d'études ont été menées pour documenter le développement des compétences dans un contexte de formation informel. Il devient donc essentiel de porter une attention aux différents contextes de formation offerts aux directions d'école compte tenu de leur rôle crucial dans la réussite des élèves (Barber, Whelan \& Clark, 2010). 


\section{But et questions de recherche}

Dans un contexte de renouvellement des directions d'école dans lequel il est ardu de recruter des candidats de qualité et dans lequel certains contextes de formations sont critiqués de même que d'autres sont peu étudiés, il s'avère essentiel de mener une étude plus approfondie, de nature qualitative dans ce domaine. Afin de connaître quels contextes de formation ont le plus aidé et peuvent éventuellement le plus aider les nouvelles directions, nous avons demandé à des acteurtrice-s de l'éducation, dans un premier temps, d'identifier quels organismes ou groupes de personnes ont le plus aidé les nouvelles directions à développer leurs compétences pour occuper un poste à la direction d'une école et, dans un deuxième temps, d'identifier quels organismes ou groupes de personnes peuvent éventuellement le plus aider les nouvelles directions à développer leurs compétences pour mieux gérer leur école.

Pour les nouvelles directions d'école, soient celles ayant cinq ans et moins de pratique, nous avons demandé 1) d'identifier quels organismes ou groupes de personnes vous ont le plus aidé à développer vos compétences pour occuper un poste à la direction d'une école et, 2) d'identifier quels organismes ou groupes de personnes peuvent éventuellement le plus vous aider à développer vos compétences pour mieux gérer votre école.

Pour éviter l'ambigüité du terme technique, contextes de formation, nous avons demandé aux participants de nommer des organismes ou des groupes de personnes. À l'aide de notre cadre conceptuel, nous avons repéré les contextes de formation en cause. Par exemple, l'université représente un contexte formel, les ateliers du district scolaire un contexte non formel et les amis un contexte informel. D’emblée, précisons que nous n'avons pas cherché à établir de liens entre les contextes de formation et les types de compétences pouvant être développés.

\section{Méthodologie}

Notre étude s'inscrit dans un cadre qualitatif et interprétatif, car elle permet de mieux comprendre le sens que les participant-e-s donnent à leurs réponses, et ce, à partir du vécu des chercheur-euse-s (Savoie-Zajc, 2011). Qui plus est, à partir des données qualitatives, nous avons effectué un traitement quantitatif, ce que Creswell (2009) appelle la transformation des données. Globalement, notre étude poursuit des buts pragmatiques et utilitaires, c'est-à-dire que l'analyse des résultats obtenus peut mener à des applications pratiques (Savoie-Zajc, 2011). En effet, par l'analyse des réponses, nous visons à identifier les contextes de formation qui siéent aux besoins spécifiques des nouvelles directions d'école.

\section{Participant-e-s}

Pour atteindre nos objectifs de recherche, nous avons cherché à rejoindre une variété de personnes du monde de l'éducation de langue française des dix 
provinces canadiennes. Ainsi, 101 acteur-trice-s de l'éducation ont été interviewés ${ }^{2}: 20$ directions générales ou adjointes de l'éducation au niveau des districts scolaires (DG+DGA), cinq président-e-s d'association de directions d'école (PA), 18 professeur-e-s d'université (PU), 28 directions d'école ayant six ans et plus d'expérience (DÉ6+) et 30 directions et directions adjointes d'école ayant cinq ans et moins d'expérience (DÉ5-). Nous appelons ces dernières, «les nouvelles directions d'école».

\section{Instrument de collecte de données}

Pour répondre aux besoins de l'étude, deux grilles d'entrevue ont été développées. Une première a été conçue pour les DÉ5- alors que la deuxième grille s'adresse aux autres participant-e-s consulté-e-s (DG+DGA, PA, PU et DÉ6+). Les grilles comprennent six sections: 1) l'identification du répondant; 2) les forces et les compétences que possèdent les DÉ5-; 3) les contextes de formation (les organismes ou groupes de personnes) qui ont aidé les DÉ5- à développer leurs compétences; 4) les compétences que les DÉ5- doivent consolider et les tâches pour lesquelles elles ont besoin d'aide; 5) les contextes de formation (les organismes ou les groupes de personnes) qui peuvent éventuellement le plus aider les DÉ5- à développer leurs compétences et 6) la priorité accordée aux différentes compétences des directions d'école. Une étude pilote a été menée auprès de quatre futures directions d'école pour ajuster les grilles d'entrevue. Enfin, les entrevues semi-dirigées (Savoie-Zajc, 2011) avec les participant-e-s se sont déroulées de l'automne 2008 au printemps 2009 dans leur milieu de travail respectif. Elles ont été enregistrées et retranscrites. Dans le cadre de cet article, seuls les résultats aux sections trois et cinq des grilles d'entrevue sont présentés.

\section{Analyse des données}

Pour traiter les données recueillies, nous avons opté pour la méthode d'analyse qualitative et inductive modérée (Savoie-Zajc, 2011). Afin de faciliter la gestion du contenu pour l'analyse des données, le logiciel NVivo 10 a été utilisé. Nous avons procédé en sept temps: 1) un premier codage en parallèle a été fait avec les verbatim de, cinq participant-e-s ayant des fonctions professionnelles différentes, et ce par la chercheuse principale et deux assistantes de recherche; 2) pour comparer les codes émergents, il y a eu une discussion entre elles; 3) à partir du consensus sur les codes, un codage de 10 autres participant-e-s a été fait par les trois « codeuses" afin d'obtenir un accord interjuge satisfaisant. Cette étape a permis de clarifier certains codes et d'ajouter ceux qui ont émergés; 4) après le codage de 50 participant-e-s par une des deux assistantes, deux rencontres entre la chercheuse et l'assistante ont eu lieu pour condenser les codes (renommer, élargir, fusionner et éliminer des codes et sous-codes); 5) une assistante a ensuite codé les autres entrevues; 6) après le codage des 101 verbatim, les quelques codes émergents ont été classifiés par la chercheuse et une assistante qui se sont rencontrées à nouveau; 7) une dernière vérification a été faite par la chercheuse. 
Quelques codes ont été modifiés par elle. Dans la section subséquente, nous présentons quelques données descriptives quantitatives bien que l'étude est d'abord et avant tout qualitative.

\section{Résultats}

Dans cette section, nous exposons les résultats aux deux questions formulées aux participants.

\section{Contextes de formation qui ont aidé Les DÉ5- à \\ développer leurs compétences pour occuper un poste de direction d'école}

Nous avons demandé aux participant-e-s de nommer quels organismes ou groupes de personnes ont aidé les DÉ5- à développer leurs compétences pour occuper un poste de direction d'école. L'analyse des entrevues montre que les réponses des participant-e-s se situent dans les trois contextes de formation. Pour cette question, le nombre total de participant-e-s est sur 93, car nous avons omis de poser la question à huit personnes : trois DÉ6+, trois DÉ5- et deux PU de notre étude.

\section{Contexte de formation formel}

Près de 61\% (57/93) des participant-e-s affirment que le contexte formel de formation a aidé les DÉ5- à développer leurs compétences. Par ailleurs, 56\% (15/27) de ces dernières sont de cet avis. Selon un PU, les DÉ5- sont:

bien outillées pour adhérer à ces postes-là du point de vue théorique, alors que dans notre temps, on découvrait au fur et à mesure. Alors que là, avec les cours qu'elles suivent, entre autres, ici à l'université et un peu partout là, la théorie elles la savent. (6d2.6)

Dans la même foulée, une autre PU avance que la formation universitaire les aide «à mieux comprendre l'univers dans lequel ils sont» $(6 \mathrm{~d} 1.12)$ et un PA mentionne qu'elle «comble leurs besoins» (6c2.4). De son côté, une DGA souligne (:)

parce que ça fait moins longtemps qu'ils sont sortis de l'université, on voit une différence lorsque quelqu'un a fait sa maitrise au niveau du leadership en général. Ils ont déjà une base qui leur permet de jouer ce rôle-là que lorsqu'ils n'ont pas nécessairement suivi cette formation-là. (9b2.1)

Selon une DÉ5-, «le cours que je fais à la maîtrise est sur la supervision, donc ça m'aide encore plus, non seulement à les superviser [les enseignant-e-s], mais à les évaluer» (2f2.2). 


\section{Contexte de formation non formel}

Ce sont 55\% (51/93) des participant-e-s de l'étude qui considèrent le contexte de formation non formel comme étant un contexte qui a aidé les DÉ5- à développer leurs compétences. Selon ces dernières, ce contexte les a aidées dans 67\% (18/27) des cas. Pour ce contexte, nous incluons d'abord, toutes les formations offertes de façon ponctuelle sous forme d'ateliers organisés par le district scolaire, le ministère de l'Éducation et l'association professionnelle. Nous considérons aussi toutes les activités coordonnées par le district scolaire telles que le mentorat, la communauté d'apprentissage professionnelle, le groupe de développement professionnel. Par ailleurs, plusieurs participant-e-s ont mentionné les rencontres planifiées avec les DG+DGA. Nous retrouvons aussi, mais citées moins souvent, les rencontres avec des surintendant-e-s, des conseillers-ères pédagogiques, l'équipe-école (direction adjointe et enseignant-e-s) voire le conseil d'école 3 . Également, nous comptons les services offerts par des organismes publics, tels que les services à la famille, les services à l'enfant. De plus, assister à des conférences provinciales et au regroupement provincial constitue des moyens pour développer les compétences des DÉ5-.

Deux DÉ5- précisent comment elles ont reçu du soutien de leur district scolaire: "Juste la formation qui nous était donnée; on avait souvent des gens au niveau du district, comme notre DG. C'était tous des gens compétents. On était entouré de gens compétents qui nous donnaient des bonnes formations» (9fl.1); «[...] j'ai eu beaucoup d'aide de mon conseil, de mon directeur des ressources humaines, [de] mon directeur général» (2f1.1). A fortiori, une DG confirme l'aide apportée par leur district aux DÉ5-:

Cette année, la plupart des [nouvelles directions d'écoles] ont vécu une expérience unique, très forte, qu'on avait appelée "Moving» and «Improving». La province [le ministère de l'Éducation] a subventionné dix projets pilotes, c'est-à-dire qu'il a regroupé dix conseils scolaires et ils ont travaillés dans un projet pilote avec un leader et nous avons conçu une formation pour ces directions d'école qui voulaient participer. On a eu neuf sur douze et elles ont pu travailler avec les grands de l'éducation au Canada. (2a2.2)

Une DÉ6+ souligne que le personnel de l'association des directions d'école les appuie beaucoup et forcément soutient les DÉ5- «[...]. Il va nous offrir de la formation et il nous appuie aussi au niveau légal. Tout ce qui est [de l'ordre de la] responsabilité légale, c'est énorme» (5e1.1). Un PU corrobore ces dires: «elles ont beaucoup de soutien de leur association» (6d2.4).

\section{Contexte de formation informel 1, 2 et 3}

En ce qui a trait au contexte de formation informel, à la lumière de nos résultats, nous avons divisé cette catégorie en trois. Le contexte informel de formation 1 regroupe les échanges que les directions d'école ont de façon spontanée avec d'autres directions d'école, des enseignants, des parents, des amis, etc. Le contexte 
informel de formation 2 rassemble des activités telles que les lectures, la consultation d'information dans Internet, l'apprentissage par «essais et erreurs» ou sur le tas, l'autoanalyse et la participation bénévole à des activités dans la communauté. Le contexte informel de formation 3 touche toutes les années d'expérience en éducation, soit comme enseignant-e, conseiller-ère pédagogique, etc.

Au total, 84\% (78/93) des participants affirment que le contexte informel de formation a aidé les DÉ5- à développer leurs compétences. Pour le contexte de formation informel 1, 23\% (21/93) des participant-e-s de l'étude dont 33\% (9/27) des DÉ5- indiquent que les autres collègues ont contribué au développement des compétences des DÉ5-. Un PU mentionne: «Elles parlent beaucoup avec leurs pairs» (7d1.1). Une DÉ5- précise: «Je pense aussi que le réseau de contacts, de collègues avec qui on travaille est aidant parce qu'on peut facilement échanger, trouver des pistes de solutions, grandir à travers ça» (6f1.4).

Il appert que 27\% (25/93) des participant-e-s de la recherche estiment que le contexte de formation informel 2 a aidé les DÉ5- à développer leurs compétences et $41 \%$ (11/27) des DÉ5- en font également état. Une DÉ6+ souligne que les compétences se développent sur le terrain: «Elles apprennent aussi beaucoup sur le tas» (7e2.2). Une DÉ5- mentionne qu'elle lit sur les domaines qu'elle connaît moins et se permet aussi de s'autoanalyser:

[...] en lisant aussi la littérature, et là je vois où j'ai une lacune, je vais voir et je dis: "Qu'est-ce que je pourrais faire pour faire mieux dans ce domaine-là?» Ça ne veut pas dire que ça se réalise, mais ça veut dire que j’y suis attentive. Je parle pour moi, mais je pense qu'il faut être autocritique, il faut s'objectiver constamment et s'observer. (2f1.3)

Pour le contexte de formation informel 3, 58\% (54/93) de l'ensemble des participant-e-s et 74\% (20/27) des DÉ5- mentionnent que ce sont les années d'expérience en éducation qui ont aidé les DÉ5- à développer leurs compétences. Une DÉ5- résume bien l'ensemble des activités de ce contexte de formation informel:

je pense que quelque part c'est l'expérience de vie comme enseignant en milieu minoritaire qui met l'esprit en éveil. Dans le sens qu'on cherche toujours comment on peut faire mieux? Comment on peut aller chercher ça pour...?

On veut aller là... Comment peut-on s'y rendre? (3f2.3)

Fait à noter, 33\% (31/93) de tous les participant-e-s mentionnent que les compétences des DÉ5- sont innées. Cette donnée n'appartient à aucun des trois contextes de formation évidemment.

\section{Contextes de formation qui peuvent éventuel- lement aider les DÉ5- à développer leurs compé- tences}

Nous avons demandé à tous les participant-e-s d'identifier quels organismes ou groupes de personnes peuvent aider éventuellement les DÉ5- à développer leurs compétences dans le cadre de leurs fonctions. L'analyse des données révèle que 
les trois contextes de formation peuvent contribuer à développer les compétences des DÉ5- dans le futur.

\section{Contexte de formation formel}

Un peu plus de 51\% (52/101) des répondant-e-s s'entendent pour dire que la formation formelle représente le contexte à favoriser pour développer éventuellement les compétences des DÉ5-. Ces dernières soulignent ce contexte à $40 \%$ (12/30). À ce sujet, deux DÉ5- soulignent que la formation universitaire est «un élément clé à considéren» (6a2.2) en leur fournissant, entre autres, "des outils pour mieux réfléchin (1d1.1). Une DÉ6+ suggère qu'

[...] au niveau de la maitrise, au niveau des universités, ils devraient investir davantage ou exiger davantage de cours pour les gens qui vivent la direction d'école, encore plus de formation au niveau de l'évaluation du personnel (2e1.1).

Un PU mentionne que les cours universitaires sont importants pour aider les nouvelles directions d'école à développer éventuellement leurs compétences, car

[...] on remarque que tous ceux qui ont suivi des cours à l'université depuis quelque temps sont beaucoup plus informés, sont beaucoup plus à l'affût [...]. Ils connaissent les choses; même s'ils sont jeunes et sans expérience, ils connaissent les choses (6d2.6).

\section{Contexte de formation non formel}

Pour 82\% (83/101) des participant-e-s, le contexte non formel de formation constitue un contexte qui peut aider les nouvelles directions à développer leurs compétences dans le futur. Elles sont $90 \%$ (27/30) des DÉ5- à affirmer la même chose. Pour la majorité des participant-e-s, le district scolaire semble être un organisme pouvant éventuellement aider les DÉ5- à développer leurs compétences, soit en offrant des sessions de formation non créditées ou en mettant en place un service d'accompagnement professionnel (mentorat, coaching). Des participant-e-s suggèrent que les DG+DGA d'un district scolaire ont un rôle important à jouer pour soutenir les DÉ5- dans le futur. D'ailleurs, une DG mentionne:

Je pense que le supérieur immédiat d'une direction d'école a aussi un rôle à ce niveau-là. Bien moi, j’ai un rôle aussi à faire lorsque je rencontre mes directions d'école. Je n'ai peut-être pas un rôle de formation, mais j'ai un rôle de questionnement qui permet peut-être à des gens de se dire «bien ça il faudrait peut-être que je creuse ça davantage, que je consulte davantage». (6a2.2)

L'association professionnelle des directions d'école a aussi été identifiée comme un organisme pouvant aider les DÉ5- dans le futur en organisant des formations selon les besoins exprimés par leurs membres. À ce sujet, une PA mentionne: "Qui de mieux placés que les gens eux-mêmes qui sont sur le terrain pour répondre aux besoins» (6c1.3). Cependant, dans certaines provinces, nous ne retrouvons 
pas d'association professionnelle des directions d'école de langue française pour soutenir les DÉ5-. D’ailleurs, une DG déplore cette situation:

S'il y avait une organisation professionnelle dans leur langue ici, [...] mais le groupe n'est pas assez nombreux, c'est une chose. Le secteur anglophone a un regroupement qui lui travaille ensemble, se donne de la formation [...]. Alors nous, on n'a pas cette chance-là. Nous, au district scolaire, [il faudra] mettre un plan en place pour appuyer nos directions, puis leur fournir de la formation. Puis, ça serait peut-être se regrouper avec des associations ou des regroupements francophones [...]. (8a2.1)

Également, une DÉ6+ affirme que «tous les partenaires, exemple les hôpitaux, les centres culturels, la communauté en général, qui sont des organismes à but non lucratif, pourraient nous aider beaucoup" (5e1.6).

\section{Contextes de formation informel 1, 2 et 3}

Près de 52\% (53/101) des participant-e-s de l'étude suggèrent que le contexte informel de formation constitue un contexte qui peut aider éventuellement les nouvelles directions à développer leurs compétences. Quant au contexte de formation informel 1, 48\% (48/101) des participant-e-s de l'étude et, plus spécifiquement, 50\% (15/30) des DÉ5- considèrent qu'il peut permettre éventuellement d'aider les DÉ5- dans leur développement professionnel. Plusieurs affirment que les collègues des DÉ5- peuvent vraiment aider, et ce, pour différentes problématiques. Somme toute, la majorité des répondant-e-s considèrent que les DÉ5- se sentent souvent seules et que le réseautage est le moyen qui peut les soutenir véritablement.

Un PA a mentionné:

La pire chose qu'on vit en direction d'école, c'est le sentiment d'isolement.

[...] On doit toujours réinventer la roue alors que la solution a déjà été trouvée tout simplement dans l'école d'à côté. Donc le besoin de réseautage, c'est un besoin important. (6c2.4)

Pour le contexte de formation informel 2, 16\% (16/101) des participant-e-s et $23 \%(7 / 30)$ des DÉ5- croient que des activités comme l'apprentissage par essais-erreurs, la lecture, l'utilisation de l'Internet, la pratique réflexive, etc. contribueront à développer les compétences des DÉ5-. D'ailleurs, deux DÉ5mentionnent: "La lecture, il faudrait lire tout le temps, il faudrait toujours être au courant; on sort de l'université et il faudrait se remettre là-dedans régulièrement, mais on sait qu'on n'a pas toujours le temps" (6f1.5) et "personnellement, je pense que la meilleure formation c'est le temps et l'expérience, les essais, les erreurs " (8f1.1). Une DG va même jusqu'à évoquer: "C'est plutôt du temps de réflexion sur leur pratique qu'ils ont vraiment besoin, pour voir ce qui ne va pas, au lieu d'aller s'asseoir à l'université pour prendre des cours» (10a2.1). 
Évidemment, nous ne retrouvons pas de référence au contexte informel de formation 3 pouvant aider éventuellement à développer les compétences des DÉ5-.

En résumé, la figure 1 présente l'ensemble des résultats quantitatifs de notre étude mettant ainsi en exergue les contextes de formation qui ont aidé et qui peuvent éventuellement aider les DÉ5- à développer leurs compétences.

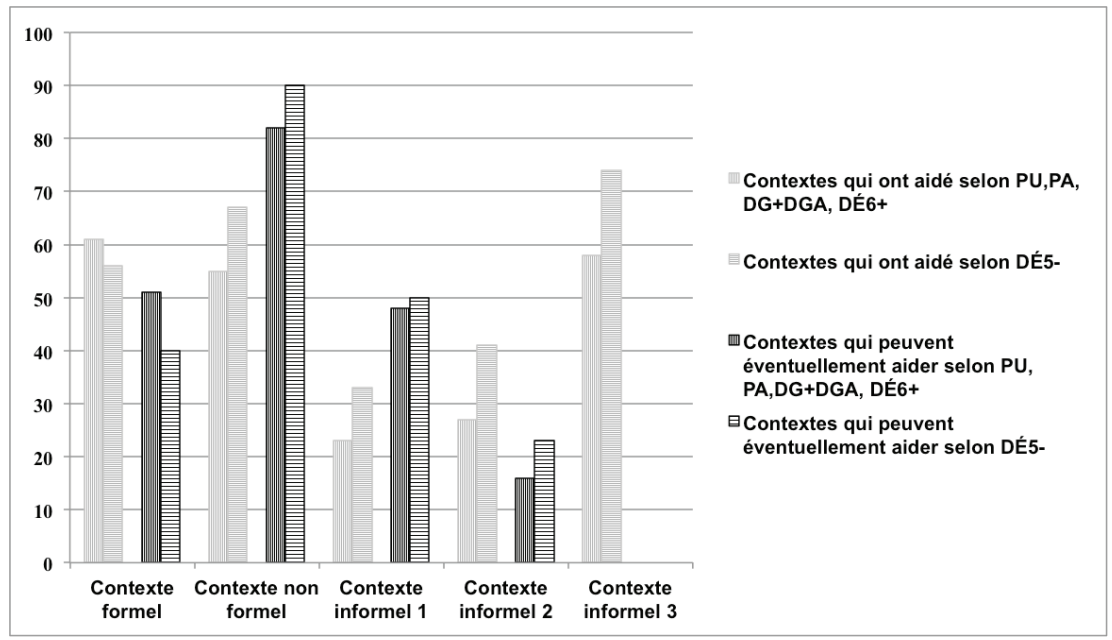

Figure 1: Proportion des participant-e-s ayant la perception que les trois contextes de formation ont aidé à développer ou peuvent aider à développer éventuellement les compétences des DÉ5-

\section{Analyse et Conclusion}

L'analyse des données révèle que, selon les PU, les PA, les DG+DGA, les DÉ6+ et les DÉ5-, le contexte de formation formel a contribué au développement des compétences des DÉ5-. Des participants indiquent que ce contexte de formation semble offrir aux DÉ5- le volet théorique nécessaire à la gestion d'une école. Même si les résultats de l'analyse quantitative d'IsaBelle et al. (2008) indiquent qu'il ne constitue pas le contexte de formation le plus apprécié par DÉ5- pour développer leurs compétences, il demeure que nos données rejoignent en partie les résultats de l'étude de Cattonar et al. (2007) qui avancent que les directions se disent très majoritairement d'accord pour dire qu'elles étaient préparées à assumer cette fonction et que leur formation antérieure, suivi dans un contexte formel, leur a été utile. En fait, comme la maitrise semble de plus en plus exigée pour accéder à un poste de direction d'école, il est possible de croire que les participants et les DÉ5- admettent la valeur des apprentissages dans le contexte 
formel de formation, mais reconnaissent aussi qu'il ne peut répondre à tous les besoins vécus in situ par les directions d'école.

Le contexte de formation non formel apparait être non seulement un contexte qui a favorisé le développement des compétences des DÉ5-, mais il constitue aussi un contexte de formation pouvant le plus les aider à développer leurs compétences dans le futur, et ce, selon la grande majorité des participant-e-s. Les formations offertes par leur district scolaire et les liens qu'elles peuvent établir avec leur direction générale du district ou leurs supérieurs semblent fort utiles. À ce sujet, nos résultats rejoignent ceux de Cattonar et al. (2007) qui montrent que près de $75 \%$ des directions de leur étude affirment être en accord avec l'énoncé J'ai reçu l'aide de mes supérieurs. Le soutien d'un supérieur qui possède une très bonne connaissance du milieu ne peut que contribuer au développement des compétences des nouvelles directions. En fait, l'appui qu'elles reçoivent peut les rassurer quant aux manières d'accomplir leurs tâches, car elles se sentent guidées par des personnes compétentes. D'ailleurs, des DÉ5-, des DÉ6+ et des DG indiquent que les DÉ5- reçoivent des ateliers de formation offerts par des formateurs compétents. Bien que nous ne retrouvions pas des associations professionnelles pour les directions d'école de langue française dans toutes les provinces, il demeure que les formations qu'elles offrent forment aussi un contexte de formation ayant contribué à développer les compétences des DÉ5-. Toujours par rapport au contexte non formel, nos résultats corroborent en partie ceux de l'étude d'IsaBelle et al. (2008) qui soulignent que les DÉ6+ et les DÉ5- apprécient développer leurs compétences en suivant des formations offertes par leur district scolaire, leur association professionnelle et le ministère de l'Éducation. Finalement, selon nos résultats, le contexte de formation non formel a aidé les DÉ5- à développer leurs compétences, mais constitue aussi le contexte qui pourra contribuer dans le futur à développer davantage leurs compétences, sans doute parce que les formations des districts scolaires offrent davantage de solutions pratiques, sur le terrain et de façon ponctuelle. Ces formations non accréditées peuvent rapidement être préparées pour répondre aux demandes spécifiques des DÉ5-.

Sanzo et al. (2011) indiquent que les cours de formation à la direction offerts conjointement entre l'université et les districts scolaires peuvent grandement bénéficier aux étudiants-tes, car ces formations combinent la théorie à la pratique. À ce sujet, les universités semblent adapter leurs programmes en administration éducationnelle afin de collaborer davantage avec le milieu scolaire (Corriveau \& Boyer, 2007). Qui plus est, la formation au principalat dans les écoles de langue française est récente, ainsi observons-nous des facultés d'éducation qui encouragent fortement certaines directions et directions générales de districts scolaires possédant des compétences spécifiques aux milieux socio-économiques faibles, au milieu minoritaire, à compléter un doctorat afin d'être engagées par les universités. Leur expertise du milieu s'avère cruciale pour la formation des futures directions d'école. 
Le contexte de formation informel 3 est souligné par les répondant-e-s et plus particulièrement par les DÉ5-comme étant un contexte qui a grandement aidé les DÉ5- à développer leurs compétences pour occuper un poste à la direction d'une école. Il semble donc normal, que les années d'expérience en éducation, in situ, soit comme enseignant-e, conseiller-ère pédagogique constituent un «contexte de formation " essentiel et très aidant pour les DÉ5- à occuper un poste à la direction. Évidemment, aucun participant n'a nommé ce contexte comme pouvant éventuellement aider les nouvelles directions à développer leurs compétences.

Le contexte informel 1 peut aussi contribuer à développer dans le futur les compétences des DÉ5+. En fait, il n'est pas étonnant que «les collègues» soient jugés comme étant des personnes pouvant aider éventuellement les DÉ5- dans leurs tâches. Nos résultats corroborent les études quantitatives d'IsaBelle et al. (2008) qui indiquent que les DÉ5- préferent développer leurs compétences dans un contexte de formation informel.

Nos résultats avancent aussi que les contextes de formation 1 et 2 ont aidé les DÉ5- a développer leurs compétences et peuvent éventuellement développer leurs compétences en communiquant avec des parents de la communauté, en participant à des activités dans la communauté et en établissant des liens avec différents organismes communautaires. De fait, la formation formelle des directions d'école aurait certainement avantage à incorporer des activités dans lesquelles elles peuvent collaborer avec leur communauté. Ainsi pouvons-nous établir un lien avec les écrits de Walker, Bryant \& Lee (2013) quant à l'importance d'opérationnaliser les programmes de formation qui siéent aux besoins locaux et à la culture locale.

Nous ne pouvons passer sous silence quelques limites de notre recherche. D'abord, les participant-e-s ont sans doute répondu sous l'effet de la désirabilité sociale. En effet, la chercheuse et les assistantes, étant du milieu universitaire, ont fort probablement influencé certain-e-s participant-e-s dans leur réponse. Également, certaines DÉ6+ vivaient une certaine difficulté à se détacher de leur vécu et répondaient en tant que nouvelles directions. De plus, des participant-e-s ont eu à se prononcer sur des formations qu'ils-elles ont peut-être suivi depuis plusieurs années, ainsi leur jugement n'est-il pas altéré avec le temps. Bien que notre étude ne visait pas à établir des liens entre les contextes de formation et les types de compétence développés, il demeure qu'une étude encore plus approfondie pourrait être menée afin de mettre en lien les contextes de formation, les composantes d'une compétence et les différentes compétences. A fortiori, d'autres études plus fines doivent être menées afin de décortiquer les contenus et les contextes de formation qui apparaissent les plus profitables aux directions d'école pour développer leurs compétences. Ces futures études, au lieu de s'appuyer sur des données de perception, pourraient plutôt collecter sur des données de performance. 


\section{Remerciements}

Les auteures tiennent à remercier les participant-e-s de la recherche, le Conseil de recherches en sciences humaines du Canada pour leur appui financier et les cochercheurs-euses Yamina Bouchamma, Paul Clarke, Lise Langlois et Claire Lapointe.

\section{Notes}

1 Au Canada, dans toutes les provinces, entre le ministère de l'Éducation et les écoles nous retrouvons un organisme qui gère les principaux dossiers éducatifs reliés aux école d'une région spécifique. Selon la province, cet organisme se nomme: district scolaire, conseil scolaire, division scolaire ou commission scolaire. Dans le cadre de notre article, nous utilisons la locution "district scolaire».

2 Le Canada est constitué de dix provinces et de trois territoires. Nous n'avons mené aucune entrevue dans les trois territoires. L'éducation est une compétence provinciale et territoriale.

3 Le Conseil d'école regroupe des parents, des enseignant-e-s et la direction.

\section{Références}

Barber, M., Whelan, F. \& Clark, M. (2010). Capturing the leadership premium: How the world's top school systems are building leadership capacity for the future. Report of Mckinsey and Company. Consulté dans http://www.mckinsey.com/clientservice/Social_ Sector/our_practices/Education/

Cattonar, B., Lessard, C., Blais, J.-G., Larose, F., Riopel, M.-C., Tardif, M. \& Bourque, J. (2007). Les directeurs et les directrices d'école au Canada: contexte, profil et travail. Enquêtes pancanadiennes auprès des directions et des enseignants d'écoles primaires et secondaires (20052006) (Rapport de recherche). Québec, Canada: Statistique Canada, Chaire de recherche du Canada sur le personnel et les métiers de l'Éducation et Centre de recherche interuniversitaire sur la formation et la profession enseignante.

Corriveau, L. \& Boyer, M. (2007). Recherche-formation: développement professionnel d'une équipe de direction d'une école secondaire au regard de la collaboration dans son milieu. Communication présentée au 75e congrès de l'ACFAS 2007, Trois-Rivières, Québec.

Creswell, J. W. (2009). Research design: Qualitative, quantitative, and mixed methods approaches (3e éd.). Thousand Oaks, CA : Sage.

Darling-Hammond, L., LaPointe, M., Meyerson, D. \& Orr, M. T. (2007). Preparing school leaders for a changing world: Executive summary. Stanford, CA: Stanford Educational Leadership Institute.

Fossey, R. \& Shoho, A. (2006). Educational leadership preparation programs in transition or in crisis? Journal of Cases in Educational Leadership, 9(3), 3-11.

Fuller, E., Young, M. \& Baker, B. D. (2011). Do principal preparation programs influence student achievement through the building of teacher-team qualifications by the principal? An exploratory analysis. Educational Administration Quarterly, 47(1), 173-216.

IsaBelle, C., Lapointe, C., Bouchamma, Y., Clarke, P., Langlois, L. \& Leurebourg, R. (2008). Contextes de formation chez les directions d'écoles francophones au Canada. Revue de recherche appliquée sur l'apprentissage, 2(1), 1-20.

LaMagdeleine, D., Maxcy, B. D., Pounder, D. G. \& Reed, C. J. (2009). The contest of university-based educational leadership preparation. In M. Young, G. Crow, J. Murphy \& R. Ogawa, (Éd.), Handbook of research on education of school leaders (pp. 129-156), New York, NY: Routledge.

Lapointe, C. (2002). Diriger l'école en milieu linguistique et culturel minoritaire. In L. Langlois \& C. Lapointe (Éd.), Le leadership en éducation. Plusieurs regards, une même passion (pp. 37-48). Montréal, Chenelière-McGraw/Hill. 
Lapointe, C. \& Gauthier, M. (2005). Le rôle des directions d'écoles dans la dynamique de la réussite scolaire. In L. DeBlois (Éd.), La réussite scolaire. Comprendre et mieux intervenir (pp. 39-50). Québec, Canada: Presses de l'Université Laval.

Lapointe, C. \& Langlois, L. (2004). L'identité professionnelle des chefs d'établissements scolaires: évolution et transformation. Éducation et francophonie, 32(2), 175-190.

Laurier, M. D. (2005). Évaluer des compétences: pas si simple... Formation et profession, Bulletin du CRIFPE, 11(1), 4-17.

Levine, A. (2005). Educating School Leaders. Consulté dans http://www.edschools.org/pdf/ Final313.pdf

Livingstone, D. W. (1999). Exploring the iceberg of adult learning: Findings of the first Canadian survey of informal learning practices (WALL Working Paper No.10). Toronto. Ontario: Centre for the Study of Education and Work, Department of Sociology and Equity Studies in Education, Ontario Institute for Studies in Education.

Marchand, L. (1997). L'apprentissage à vie. La pratique de l'éducation des adultes et de l'andragogie. Montréal, Canada: Éditions Chenelière /McGraw-Hill.

Meirieu, P. (2005). Si la compétence n'existait pas, il faudrait l'inventer... Consulté dans http:// www.meirieu.com/ARTICLES/SUR\%20LES\%20COMPETENCES.pdf

Merriam, S. B., Caffarella, R. S. \& Baumgartner, L. M. (2009). Learning in adulthood: A comprehensive guide ( $3 \mathrm{e}$ éd.). San Francisco: Jossey-Bass.

Ministère de l'Éducation, du Loisir et du Sport [MELS]. (2007). Programme de formation de l'école québécoise. Un programme de formation pour le XX1e siècle. Consulté dans http:// www.mels.gouv.qc.ca/sections/programmeFormation/secondaire2/medias/1-pfeq_chap1. pdf

Organisation de coopération et de développement économiques [OCDE]. (juin, 2008). Améliorer la direction des établissements scolaires. Repères pour l'élaboration des politiques. Direction de l'éducation, Division des politiques d'éducation et de formation, OCDE. Consulté dans http://www.oecd.org/fr/edu/apprendre-au-dela-de-l-ecole/46165252.pdf

Pounder, D. G. (2011). Leader preparation special issue: Implications for policy, practice and research. Educational Administration Quarterly, 47(1): 258-267.

Roegiers, X. \& De Ketele, J.-M. (2000). Une pédagogie de l'intégration: compétences et intégration des acquis dans l'enseignement. Bruxelles, Belgique: De Boeck Université.

Sanzo, K. L., Myran, S. \& Clayton J. K. (2011). Building bridges between knowledge and practice. Journal of Educational Administration, 49(3), 292-312.

Savoie-Zajc, L. (2011). La recherche qualitative/interprétative en éducation. In T Karsenti \& L. Savoie-Zajc (Éd.). La recherche en éducation: étapes et approches (4e éd., pp.123-147). Ville Saint-Laurent, Québec: Éditions du Renouveau Pédagogique.

Schugurensky, D. (2000). The forms of informal learning: Towards a conceptualization of the field (NALL Working Paper \#19-2000). Consulté dans http://www.nall.ca/res/19formsofinformal.htm

Seashore Louis, K., Dretzkekl, B. \& Wahlstrom, K. (2010). How does leadership affect student achievement? Results from a national US survey. School Effectiveness and School Improvement, 21(3), 315-336.

Service Canada. (2012). Directeurs d'école et administrateurs au primaire et au secondaire. Gouvernement du Canada. Consulté dans http://www.servicecanada.gc.ca/fra/qc/emploi_ avenir/statistiques/0313.shtml

Walker, A., Bryant, D. \& Lee, M. (2013). International patterns in principal preparation: commonalities and variations in pre-service programmes. Educational Management Administration and Leadership, 41(4) 405-434.

Werquin, P. (2010). Reconnaître l'apprentissage non formel et informel. Résultats, Politiques et Pratiques (rapport). Paris, France: Éditions OCDE. Consulté dans http://www.cicic.ca/ docs/oecd/rnfil.fr.pdf 
Young, M. D., Crow, G., Orr, M. T., Ogawa, R. \& Creighton, T. (2006). An educative look at «educating school leaders» UCEA, AERA \& NCPEA. Consulté dans http://www.ucea.org/ pdf/EducLeadersRespMar18.pdf.

Mots-clés: Contextes de formation formel, non formel ou informel, compétences de direction direction d'établissement scolaire, formation des directeurs d'école, école de langue française Canada.

\section{Formelle, nicht-formelle und informelle Bildungskontexte: Kompetenzentwicklung für Schulleitungen französischer Sprache in Kanada}

\section{Zusammenfassung}

Zur Weiterentwicklung von Kompetenzen neuer Schulleitungen stellen wir fest, dass neue von Universitäten, Schulbezirken o.ä. angebotene Bildungsprogramme entstanden sind. Ziel unserer Untersuchung ist es, zu identifizieren, welche Bildungskontexte (formell, nicht-formell oder informell) neuen Schulleitungen bei der Entwicklung ihrer Kompetenzen: (a) am besten geholfen haben und (b) welche ihnen künftig am besten helfen können. Im Rahmen unserer qualitativen Studie wurden 101 Akteurinnen und Akteure des Bildungsbereiches befragt. Aus den Ergebnissen geht hervor, dass alle drei Bildungskontexte (formell, nichtformell und informell) zur Entwicklung der Kompetenzen neuer Schulleitungen beigetragen haben. Zudem zeigte sich, dass Im Vergleich zu den formellen und informellen Bildungskontexten der nicht-formelle Bildungskontext (Workshops und Unterstützung von seiten des Schulbezirkes) am meisten zur Entwicklung von Kompetenzen neuer Schulleitungen beigetragen hat.

Schlagworte: Formelle, nicht-formelle und informelle Bildungskontexte, Schulleitungsompetenzen, Schulleitungsausbildung, französischsprachige Schulen Kanada

\section{Apprendimento formale, non formale o informale: sviluppo delle competenze delle direzioni scolastiche francofone in Canada}

\section{Riassunto}

Per sviluppare le competenze dei nuovi direttori di istituto abbiamo potuto rilevare la nascita di programmi formativi proposti da università, distretti scolastici, etc. Lo scopo del nostro studio è stato di identificare il contesto educativo formale, non formale o informale che 1) abbia aiutato maggiormente 2) possa eventualmente aiutare i nuovi direttori a sviluppare le loro competenze. 101 attori del mondo dell'educazione sono stati interrogati all'interno di una cornice 
di riferimento qualitativa. I risultati mostrano che i tre contesti di formazione (formale, non formale e informale) sembrano aver contribuito allo sviluppo delle competenze dei nuovi direttori. Il contesto non formale, specialmente gli ateliers e il sostegno del distretto scolastico, sembra essere quello che aiuta di più i nuovi direttori a sviluppare le loro competenze.

Parole chiave: Apprendimento formale, non formale e informale, competenze delle direzioni scolastiche, direzione d'istituto, formazione dei direttori scolastici, scuola francofona in Canada

\section{Formal, non-formal and informal education: Competency development of French speaking school principals in Canada}

\section{Summary}

To enhance the competencies of new school principals, we see the emergence of training programs proposed by universities, school districts, etc. The goal of our study is to identify which educational contexts (formal, non-formal and informal) (a) have helped the most, and (b) can best help new school principals to develop their leading competencies. A total of 101 educational agents were interviewed in this qualitative research project. Results show that the three educational contexts (formal, non-formal and informal) have contributed to develop new principals' school leading competencies. Furthermore, the non-formal education context (i.e. workshops and support from the school district) appears to be the one that could best help them to further develop their competencies.

Keywords: Formal, non-formal and informal education, School leading competencies, School principals, School leading training, French speaking school in Canada. 
